\title{
CURVAS LOGOAUDIOMÉTRICAS UTILIZANDO SENTENÇAS COMO ESTÍMULO
}

\author{
The performance-intensity function for sentences \\ with the Portuguese Sentence List Test
}

Nilvia Herondina Soares Aurélio ${ }^{(1)}$, Maristela Júlio Costa ${ }^{(2)}$

\begin{abstract}
RESUMO
Objetivo: determinar curvas logoaudiométricas, utilizando o teste Listas de Sentenças em Português (LSP), em um grupo de indivíduos normo-ouvintes e um grupo de indivíduos com perda auditiva neurossensorial, e comparar os resultados obtidos nestes grupos. Métodos: utilizou-se o teste Listas de Sentenças em Português (LSP) para determinar os Limiares de Detecção de Voz (LDV), Limiares de Reconhecimento de Sentenças no Silêncio (LRSS), e os Índices Percentuais Máximos de Reconhecimento de Sentenças no Silêncio (IPMRSS), em um grupo de indivíduos normo-ouvintes (grupo A) e um grupo de indivíduos com perda auditiva neurossensorial (grupo B). Resultados: o grupo $A$ foi composto por 19 indivíduos do sexo feminino e 11 do sexo masculino, com idades entre 18 e 40 anos. No grupo B, 15 indivíduos eram do sexo feminino e 14 eram do sexo masculino, com idades variando entre 23 e 81 anos. Para o grupo A, encontraram-se valores médios de 16,47 dB para o LDV; $23,77 \mathrm{~dB}$ para o LRSS e de $31,23 \mathrm{~dB}$ para o IPMRSS. Já no grupo $\mathrm{B}$, os valores encontrados foram de 38,38 dB para o LDV; 50,35 dB para o LRSS e 59,65 dB para o IPMRSS. Na análise estatística, encontrou-se diferença estatisticamente significante entre os grupos. Conclusão: foram traçadas as curvas logoaudiométricas para os grupos estudados. Os dados permitem inferir que a cada $1 \mathrm{~dB}$ de variação, na intensidade de apresentação das sentenças, ocasionou uma mudança de $6,77 \%$ no reconhecimento de fala para os indivíduos normo-ouvintes e de $4,70 \%$ e no grupo de indivíduos com perda auditiva.
\end{abstract}

DESCRITORES: Audição; Testes de Discriminação da Fala; Inteligibilidade da Fala; Percepção da Fala

\section{INTRODUÇÃO}

A comunicação é para o ser humano um ato primordial na vida e é por meio da audição que recebemos os estímulos necessários para que esta se estabeleça. Sabe-se, porém, que não basta ouvir para que se possa estabelecer a comunicação; faz-se necessário o reconhecimento dos estímulos acústicos ouvidos ${ }^{1}$.

(1) Fonoaudióloga do Projeto Promoção da Saúde Auditiva da Universidade Federal de Santa Maria, UFSM, Santa Maria, RS; Mestranda em Distúrbios da Comunicação Humana pela Universidade Federal de Santa Maria.

(2) Fonoaudióloga; Professora Adjunta do Departamento de Fonoaudiologia da Universidade Federal de Santa Maria, UFSM, Santa Maria, RS; Doutora em Ciências dos Distúrbios da Comunicação Humana pela Universidade Federal de São Paulo.

Conflito de interesses: inexistente
A compreensão da fala permite ao homem uma comunicação eficaz com seus semelhantes e, por ser uma habilidade tão significativa para o indivíduo, é que uma das queixas mais encontradas na rotina clínica é, justamente, a dificuldade de compreender a fala ${ }^{2}$.

Desta maneira, a habilidade para compreender a fala deve ser considerada como o aspecto mais importante a ser mensurado na função auditiva humana, pois permite avaliar a função comunicativa receptiva, fornecendo dados de como o sujeito funciona em situações de escuta diária, por meio de informações objetivas, facilmente quantificáveis ${ }^{1}$.

Na prática clínica audiológica, são aplicados testes de reconhecimento de fala com distintos tipos de estímulos, como sílabas, listas de palavras com e sem sentido, monossilábicas, dissilábicas, entre outros. No entanto, deve-se ter a preocupação em medir a capacidade de reconhecimento da fala em situações mais próximas às reais ${ }^{3}$. 
Testes audiológicos que empregam sentenças como estímulo oferecem uma aproximação direta com situações de comunicação do dia-a-dia e fornecem informações que vão orientar a conduta mais adequada a ser indicada para o indivíduo com queixa de distúrbio da comunicação, além de avaliar a real habilidade auditiva do paciente ${ }^{3}$.

A avaliação em campo livre é a forma mais fidedigna para reproduzir um ambiente no qual há necessidade de reconhecer uma mensagem falada, pois o material e a situação de teste permitem avaliar a situação binaural. Tal situação reflete a condição na qual a comunicação diária se estabelece ${ }^{4,5}$.

Com a finalidade de fornecer informações sobre a real habilidade de reconhecimento da mensagem falada em situações mais próximas as da vida diária, Costa (1998) desenvolveu o teste Listas de Sentenças em Português.O procedimento foi gravado em $C D$ que contém oito listas de sentenças foneticamente balanceadas e um ruído com espectro de fala, ambos gravados em canais independentes, permitindo a apresentação das sentenças tanto no silêncio, quanto no ruído, com intensidades de apresentação diferentes ${ }^{3}$.

O teste de Listas de Sentenças em Português (LSP) proporciona precisão e objetividade para mensurar habilidades de reconhecimento de fala de um ouvinte, como um reflexo de seu desempenho, em situações auditivas realistas e seus achados são de extrema importância para um diagnóstico clínico mais preciso ${ }^{6}$.

Várias pesquisas já foram desenvolvidas com o uso deste teste ${ }^{2,6-8}$, fornecendo informações de extrema relevância na área da audiologia clínica. Porém, alguns questionamentos relativos ao reconhecimento de fala ainda não foram respondidos por estas pesquisas. Assim, surgiu o interesse em realizar este estudo.

O presente estudo tem como objetivos determinar as curvas logoaudiométricas, utilizando o teste Listas de Sentenças em Português (LSP) ${ }^{3}$ em um grupo de indivíduos normo-ouvintes e em um grupo de indivíduos com perda auditiva neurossensorial e comparar os resultados obtidos nestes grupos.

\section{MÉTODOS}

Este estudo foi realizado no Laboratório de Próteses Auditivas (LPA) do Serviço de Atendimento Fonoaudiológico (SAF) da Universidade Federal de Santa Maria (UFSM), a partir do projeto "Pesquisa e Base de Dados em Saúde Auditiva", registrado no Gabinete de Projetos do centro de Ciências da Saúde sob o no 019731.

As avaliações foram efetuadas no período compreendido entre setembro de 2008 e março de 2009.
Os critérios de inclusão adotados foram: idade superior a 18 anos e limiares audiométricos dentro dos padrões de normalidade ou indicativos de perda auditiva neurossensorial de graus leve a moderadamente-severo ${ }^{9}$, com Limiares de Reconhecimento de Fala (LRF) inferiores a 65 dB NA, na melhor orelha.

Os critérios de exclusão adotados foram: presença de alterações neurológicas e/ou de fluência verbal, presença de presença de rolha de cerume ou de outras alterações no meato acústico externo, capazes de alterar o desempenho no teste; ausência de resposta ou dificuldade para memorizar as sentenças do teste Listas de Sentenças em Português ${ }^{3}$.

Os voluntários somente foram avaliados depois de terem concordado com a realização dos procedimentos necessários para a execução da pesquisa e assinado o Termo de Consentimento Livre e Esclarecido, após terem recebido maiores esclarecimentos sobre o objetivo e a metodologia do estudo proposto.

Antes da realização das avaliações, foi efetuada uma anamnese a fim de fornecer informações referentes a dados pessoais, queixas auditivas, história otológica, hábitos de vida diária, profissão e nível de escolaridade dos sujeitos estudados.

Em seguida, os indivíduos passaram por uma avaliação audiológica básica, na qual foi realizada a inspeção visual do meato acústico externo; audiometria tonal liminar por via aérea, nas frequências de 250 a $8.000 \mathrm{~Hz}$ e por via óssea, nas frequências de 500 a $4.000 \mathrm{~Hz}$; pesquisa do LRF, com palavras dissilábicas e pesquisa do índice percentual de reconhecimento de fala (IPRF).

As curvas logoaudiométricas foram obtidas, utilizando-se o teste Listas de Sentenças em Português ${ }^{3}$, constituído de 25 sentenças $(1 \mathrm{~A})$, outras sete listas com dez sentenças (Listas 1B, 2B, 3B, $4 \mathrm{~B}, 5 \mathrm{~B}, 6 \mathrm{~B}, 7 \mathrm{~B})$ e um ruído com espectro de fala. As sentenças e o ruído estão gravados em $C D$, em canais independentes, e foram apresentados através de um CD Player, acoplado a um audiômetro, da marca Damplex, modelo DA 65, com sistema de amplificação para audiometria em campo livre. As listas de sentenças que foram utilizadas para a realização desta pesquisa, estão descritas na Figura 1.

As sentenças foram apresentadas em campo livre, permitindo avaliar as duas orelhas, simultaneamente.

Para determinar as curvas logoaudiométricas, foi realizada a pesquisa dos Limiares de Detecção de Voz (LDV), dos Limiares de Reconhecimento de Sentenças no Silêncio (LRSS) e dos Índices Percentuais Máximos de Reconhecimento de Sentenças no Silêncio (IPMRSS). 


\section{LISTA $1 A$}

1. Não posso perder o ônibus.

2. Vamos tomar um cafezinho.

3. Preciso ir ao médico.

4. A porta da frente está aberta.

5. A comida tinha muito sal.

6. Cheguei atrasado para a reunião.

7. Vamos conversar lá na sala.

8. Depois liga pra mim.

9. Esqueci de pagar a conta.

10. Os preços subiram ontem.

11. O jantar está na mesa.

12. As crianças estão brincando.

13. Choveu muito neste fim-de-semana.

14. Estou morrendo de saudade.

15. Olhe bem ao atravessar a rua.

16. Preciso pensar com calma.

17. Guardei o livro na primeira gaveta.

18. Hoje é meu dia de sorte.

19. O sol está muito quente.

20. Sua mãe acabou de sair de carro.

\section{LISTA 1B}

1. O avião já está atrasado.

2. O preço da roupa não subiu.

3. O jantar da sua mãe estava bom.

4. Esqueci de ir ao banco.

5. Ganhei um carro azul lindo.

6. Ela não está com muita pressa.

7. Avisei seu filho agora.

8. Tem que esperar na fila.

9. Elas foram almoçar mais tarde.

10. Não pude chegar na hora.

\section{LISTA 2B}

1. Acabei de passar um cafezinho.

2. A bolsa está dentro do carro.

3. Hoje não é meu dia de folga.

4. Encontrei seu irmão na rua.

5. Elas viajaram de avião.

6. Seu trabalho estará pronto amanhã.

7. Ainda não está na hora.

8. Parece que agora vai chover.

9. Esqueci de comprar os pães.

10. Ouvi uma música linda.
LISTA 3B
1. Ela acabou de bater o carro.
2. É perigoso andar nessa rua.
3. Não posso dizer nada.
4. A chuva foi muito forte.
5. Os preços subiram na segunda.
6. Esqueci de levar a bolsa.
7. Os pães estavam quentes.
8. Elas já alugaram uma casa na praia.
9. Meu irmão viajou de manhã.
10. Não encontrei meu filho.

\section{LISTA 4B}

1. Sua mãe pôs o carro na garagem.

2. O aluno quer assistir ao filme.

3. Ainda não pensei no que fazer.

4. Essa estrada é perigosa.

5. Não paguei a conta do bar.

6. Meu filho está ouvindo música.

7. A chuva inundou a rua.

8. Amanhã não posso almoçar.

9. Ela viaja em dezembro.

10. Você teve muita sorte.

\section{LISTA 5B}

1. Depois, a gente conversa.

2. Ela acabou de servir o almoço.

3. Esta carta chegou ontem.

4. Preciso terminar o meu trabalho.

5. Não posso esquecer da mala.

6. A rua estava muito escura.

7. A data do exame foi adiada.

8. Elas alugaram um carro no verão.

9. Minha viagem foi ótima.

10. Eles foram comprar pães.

\section{LISTA 6B}

1. Vou viajar as nove da manhã.

2. Meu irmão bateu o carro ontem.

3. Prometi a ele não contar o segredo.

4. Cheguei atrasada na aula.

5. Esta rua é perigosa.

6. Esqueci da bolsa na sua mesa.

7. Ela comprou os últimos pães.

8. A casa de campo já foi alugada.

9. Os preços não devem subir.

10. Não falei com sua filha.

Figura 1 - Listas de Sentenças em português utilizadas

Para estabelecer a intensidade de apresentação das sentenças, foi usado como referência um tom puro gravado no canal do CD onde estão gravadas as sentenças, pois o sinal de fala é um som complexo, que apresenta uma variação de 30
$\mathrm{dB}$ entre o som mais intenso e o menos intenso, oscilando $12 \mathrm{~dB}$ acima e $18 \mathrm{~dB}$ abaixo da média, necessitando, portanto de um som contínuo de referência para manter sempre as mesmas condições de apresentação ${ }^{10}$. 
A saída de cada canal do CD foi calibrada através do VU-meter do audiômetro antes do início da avaliação de cada indivíduo. O tom de $1 \mathrm{kHz}$ presente no mesmo canal do CD em que estão gravadas as sentenças foi colocado no nível zero.

Todos os procedimentos foram realizados em uma cabina acusticamente tratada, com o paciente posicionado de frente, a um metro em relação à caixa acústica, com o sinal de fala (sentenças) sendo sempre apresentado a $0^{\circ}$ Azimute, ou seja, formando um ângulo de 0 ㅇ em relação aos planos: horizontal e vertical. Essa posição é a mais vantajosa, pois permite maior precisão nas medidas e conforto ao paciente, além de ser a que mais se aproxima de uma situação de conversação normal ${ }^{4}$.

Foi realizada previamente, por um profissional habilitado para este serviço, registrado no Inmetro $\mathrm{SP}$, a calibração do equipamento; porém, considerando as características do sinal de teste e da necessidade de manter sempre as mesmas condições acústicas do ambiente durante toda a pesquisa, as medidas em campo livre, foram monitoradas pela examinadora com o auxílio de um Medidor de Pressão Sonora Digital, da marca Radio Shack.

A escala utilizada foi a Escala $A$, com respostas rápidas, por ser considerada a que melhor se aproxima da resposta auditiva humana, além de ser a mais usada pela maioria dos pesquisadores nesta área ${ }^{2,7,11}$.

A primeira medida obtida foram os Limiares de Reconhecimento de Sentenças no Silêncio (LRSS), que consiste na intensidade necessária para o indivíduo reconhecer em torno de $50 \%$ dos estímulos apresentados, neste caso, as sentenças de uma lista de dez.

Porém, anteriormente à obtenção do LRSS, foi realizado um treinamento, que consistiu na apresentação das dez primeiras sentenças da lista $1 \mathrm{~A}$, na condição de escuta binaural, no silêncio.

Este treinamento foi importante, pois possibilitou a familiaridade do paciente com o teste e permitiu a obtenção dos níveis de intensidade necessários para que cada indivíduo reconhecesse corretamente a primeira sentença de cada lista a ser apresentada a seguir. Assim sendo, a intensidade inicial de apresentação da primeira sentença de cada lista do teste propriamente dito, foi baseada nos resultados encontrados no treinamento anteriormente descrito.

A técnica para apresentação das sentenças foi baseada na estratégia denominada sequencial ou adaptativa ou, ainda, ascendente-descendente ${ }^{12}$.

Quando o indivíduo respondia corretamente, diminuía-se a intensidade de apresentação do estímulo seguinte. Quando a resposta era incorreta, aumentava-se a intensidade de apresentação do estímulo seguinte. Uma resposta só foi considerada correta, quando o indivíduo repetiu, sem nenhum erro ou omissão, toda a sentença apresentada.

Os intervalos utilizados até a primeira mudança no tipo de resposta, ou seja, quando o paciente não respondeu corretamente uma sentença, foram de 4 $\mathrm{dB}$ e, posteriormente, os intervalos de apresentação dos estímulos foram de $2 \mathrm{~dB}$ até o final da lista.

A média dos valores foi calculada a partir do nível de apresentação em que ocorreu a primeira mudança no tipo de resposta, até o valor de apresentação da última sentença da lista. 1B.

Para a obtenção dos LRSS foi utilizada a lista

A seguir, foram iniciados os procedimentos para a obtenção dos Índices Percentuais Máximos de Reconhecimento de Sentenças no Silêncio (IPMRSS), ou seja, a intensidade necessária para o indivíduo reconhecer $100 \%$ das sentenças de uma lista apresentada.

Para a obtenção dos índices, de modo que o exame não fosse muito extenso e cansativo ao paciente, primeiramente, foram somados $4 \mathrm{~dB}$ ao valor encontrado na pesquisa do LRSS. Nesta intensidade, uma lista de sentenças foi apresentada. Ao final da lista, se o paciente não respondesse todas as frases corretamente, acrescentava-se $4 \mathrm{~dB}$ na intensidade e uma nova lista de sentenças era apresentada. Este procedimento foi repetido até o indivíduo reconhecer todas as sentenças da lista, ou seja, obter $100 \%$ de reconhecimento.

Ao obter $100 \%$ de acerto, a fim de verificar se o indivíduo não teria o mesmo desempenho em uma intensidade menor, eram subtraídos $2 \mathrm{~dB}$ da intensidade onde se obteve o índice de $100 \%$ de reconhecimento e uma nova lista era apresentada.

Para fins de análise estatística e apresentação dos resultados, os dados obtidos na pesquisa do IPMRSS foram representados, não pela porcentagem de reconhecimento, mas sim pela intensidade de apresentação das sentenças. Também, foi utilizada somente a intensidade em que o indivíduo foi capaz de reconhecer $100 \%$ dos estímulos apresentados; as intensidades nas quais foram obtidos os demais índices foram desconsideradas.

As listas 2B a 6B, contendo dez sentenças cada, foram utilizadas na pesquisa dos IPMRSS.

Posteriormente, a intensidade de apresentação das sentenças foi diminuída para níveis nos quais o indivíduo referisse não ouvir absolutamente nada dos estímulos de fala; então o estímulo foi apresentado em níveis de intensidade crescente de 1 em $1 \mathrm{~dB}$, até que o indivíduo fosse capaz apenas de detectar o estímulo sonoro, obtendo-se assim, o 
Limiar de Detecção de Voz (LDV). As listas utilizadas para esse procedimento foram 1A e 7B.

Os resultados foram expressos em dB NPS na escala A (dB (A) NPS).

Os indivíduos avaliados foram distribuídos em dois grupos: indivíduos normo-ouvintes (Grupo A) e indivíduos com perda auditiva neurossensorial (Grupo B).

Para a análise e descrição dos resultados dos indivíduos com perda auditiva, foram considerados apenas os resultados da melhor orelha, pois a ela estão correlacionados os resultados obtidos em uma avaliação em campo livre.

Este estudo foi aprovado pela Comissão de Ética em Pesquisa (CEP) da UFSM em 05/12/2006, protocolo $\mathrm{n}^{\circ}$ 23081.0.016862/2006-09.

Os resultados foram analisados inicialmente por meio de estatística descritiva e para realizar a comparação entre os grupos em cada variável, foi utilizado o teste $U$ de Mann-Whitney, visto que os dados não apresentaram aderência à curva normal ${ }^{13}$.

O nível de rejeição para a hipótese de nulidade foi fixado em um valor menor ou igual a 0,05 (5\%).

\section{RESULTADOS}

Foram avaliados 59 indivíduos, sendo 30 pertencentes ao grupo $\mathrm{A}$ e 29 pertencentes ao grupo $\mathrm{B}$.

O grupo A foi composto por 19 indivíduos do sexo feminino e 11 do sexo masculino, com idades entre 18 e 40 anos. No grupo B, 15 indivíduos eram do sexo feminino e 14 eram do sexo masculino, com idades variando entre 23 e 81 anos.

As médias aritméticas, os valores máximos e mínimos e os desvios padrão para as variáveis LDV, LRSS e IPMRSS dos grupos A e B, assim como a análise realizada com 0 teste de $U$ de Mann-Whitney, a fim de comparar os resultados obtidos entre os grupos, estão expostos na Tabela 1.
Todos os resultados estatisticamente significantes foram assinalados com um asterisco $\left({ }^{*}\right)$.

$\mathrm{Na}$ Figura 2 estão expostas as curvas logoaudiométricas obtidas a partir das avaliações realizadas com o teste LSP.

\section{DISCUSSÃO}

Neste estudo, utilizou-se o teste Listas de Sentenças em Português para obtenção de curvas logoaudiométricas utilizando sentenças como estímulo.

As curvas logoaudiométricas (Figura 2) foram constituídas pelos níveis médios de intensidade necessários para que os indivíduos detectassem (LDV) ${ }^{14}$, reconhecessem aproximadamente 50\% (LRSS) ${ }^{12}$ e reconhecessem 100\% ${ }^{3,15}$ (IPMRSS) das sentenças apresentadas em listas contendo dez sentenças cada.

Apesar de avaliar os indivíduos em campo livre não fazer parte da rotina clínica, principalmente indivíduos com audição normal, é importante estabelecer a intensidade média de estímulo de fala necessária para que um indivíduo seja capaz de estabelecer uma comunicação satisfatória na sua vida diária, pois assim pode ser feita uma análise mais apurada de como os indivíduos que apresentam perda de audição vão se comportar em uma situação semelhante.

Neste estudo, a pesquisa foi iniciada com medidas obtidas no silêncio, a fim de se estabelecer a capacidade de reconhecimento de fala do indivíduo, primeiramente, em situação favorável de escuta. Pretende-se, em outro momento, a realização de um estudo em situação desfavorável, ou seja, na qual o ruído competitivo esteja presente, pois neste ambiente, no qual o número de pistas cai significativamente, o sujeito pode apresentar outras dificuldades que podem interferir na compreensão da fala.

Portanto, procurou-se primeiro conhecer estes valores, no silêncio, em indivíduos adultos audiologicamente normais e, posteriormente, em um grupo

Tabela 1 - Médias aritméticas, valores máximos e mínimos, desvios padrão e resultados da comparação entre os grupos realizada através do teste U de Mann-Whitney

\begin{tabular}{ccccccc}
\hline Variáveis & Grupo & Média & Mín & Máx & DP & Diferença \\
\hline LDV & A & 16,47 & 12,00 & 22,00 & 2,74 & \multirow{2}{*}{$0,0027^{*}$} \\
& B & 38,38 & 23,00 & 60,00 & 9,27 & \\
\hline \multirow{2}{*}{ LRSS } & A & 23,77 & 19,60 & 31,50 & 2,89 & \multirow{2}{*}{$0,0009^{*}$} \\
& B & 50,35 & 26,70 & 71,00 & 10,23 & \\
\hline IPMRSS & A & 31,23 & 24,00 & 37,00 & 3,06 & \multirow{2}{*}{$0,0022^{*}$} \\
& B & 59,65 & 37,00 & 85,00 & 11,15 & \\
\hline
\end{tabular}

$\left(^{*}\right)$ Existe correlação estatisticamente significante $(p<0,05)$ 


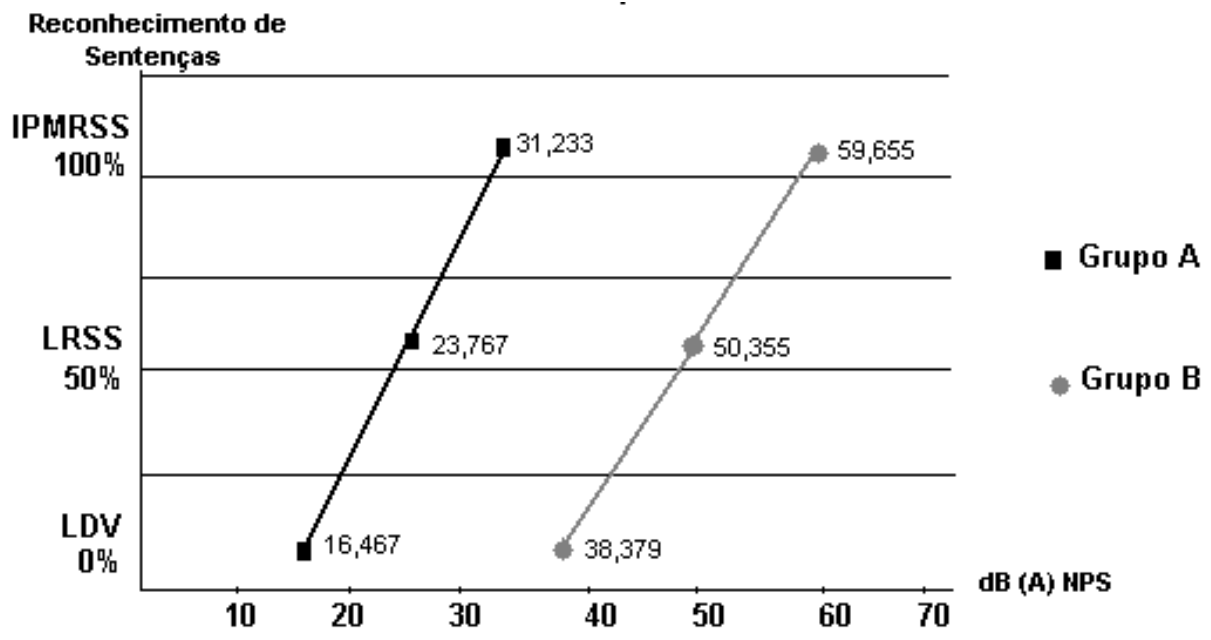

Figura 2 - Curvas Logoaudiométricas obtidas através dos valores médios em dB (A) NPS, encontrados na pesquisa dos Limiares de Detecção de voz (LDV), dos Limiares de Reconhecimento de Sentenças no Silêncio (LRSS) e dos Índices Percentuais de Reconhecimento de Sentenças no Silêncio (IPMRSS), através do teste Listas de Sentenças em Português (LSP- Costa, 1998) nos dois grupos estudados

de indivíduos com perda auditiva neurossensorial, e então foram comparados os valores obtidos nos dois grupos (Tabela 1).

Para os indivíduos normo-ouvintes (grupo $A$ ), foram encontrados valores médios de 16,47 dB (A) NPS para o LDV; 23,77 dB (A) NPS para o LRSS e de $31,23 \mathrm{~dB}(\mathrm{~A})$ NPS para o IPMRSS.

Assim sendo, foi verificado que os indivíduos que apresentam limiares tonais menores ou iguais a 25 dB NA, são capazes de reconhecer $50 \%$ de uma conversação em campo livre, em ambiente silencioso, em uma intensidade em torno de 23,77 dB (A) NPS, e 100\% dos estímulos em uma intensidade aproximada de 31,23 dB (A) NPS, ou seja, bem abaixo da intensidade média da fala que é 60-65 dB NPS ${ }^{16}$, o que possibilita que os indivíduos sejam capazes de reconhecer voz cochichada, distante, sem necessitar de contexto ou apoio visual, ou ainda estabelecer uma comunicação satisfatória com interlocutores cuja pronúncia não seja muito clara, como é comum no nosso dia-a-dia.

Ao analisar os intervalos entre os três pontos da curva, percebe-se uma diferença de 7,30 dB (A) NPS entre o valor médio do LDV e do LRSS, e uma diferença de 7,46 dB (A) NPS entre o LRSS e o IPMRSS, portanto o intervalo entre o valor médio do LDV e do IPMRSS foi de $14,76 \mathrm{~dB}$ (A) NPS.

Pode-se inferir por intermédio dos dados encontrados, que basta um acréscimo de aproximadamente $7 \mathrm{~dB}$ para que os indivíduos normo-ouvintes passem do nível de detecção sonora ao nível liminar. Da mesma forma, com um novo acréscimo de aproximadamente $7 \mathrm{~dB}$ os indivíduos normo-ouvin- tes passam do nível liminar à compreensão total do discurso apresentado.

Considerando que $14,76 \mathrm{~dB}$ representaram o intervalo aproximado de 0 a $100 \%$ de reconhecimento de sentenças, pode-se afirmar que cada 1 $\mathrm{dB}$ foi responsável por $6,77 \%$ de mudança na inteligibilidade da fala.

Por sua vez, os indivíduos com perda auditiva neurossensorial (grupo B), obtiveram valores de $38,38 \mathrm{~dB}$ para o LDV; $50,35 \mathrm{~dB}$ para o LRSS e $59,65 \mathrm{~dB}$ para o IPMRSS.

Os intervalos entre os pontos da curva foram de $11,97 \mathrm{~dB}$ entre o valor médio do LDV e do LRSS; de $9,30 \mathrm{~dB}$ entre o LRSS e o IPMRSS; e de $21,28 \mathrm{~dB}$ (A) NPS entre o valor médio do LDV e do IPMRSS.

Observa-se, que foram necessários aproximadamente $12 \mathrm{~dB}$ para os indivíduos com perda auditiva saírem do nível de detecção sonora e passarem ao nível do limiar. E, cerca de $9 \mathrm{~dB}$ acima do limiar para alcançarem o máximo reconhecimento.

O intervalo aproximado de 0 a $100 \%$ de reconhecimento de sentenças foi constituído por 21,28 dB. Portanto, para os indivíduos com perda auditiva neurossensorial, pode-se considerar que a cada 1 $\mathrm{dB}$ de mudança na intensidade do discurso, ocorre uma mudança de $4,70 \%$ no reconhecimento de fala.

Como era de se esperar, foi observado que para os indivíduos do grupo B, que apresentam perda auditiva neurossensorial, houve a necessidade de mais intensidade para se obter as três medidas pretendidas.

O valor médio encontrado para o LDV nos indivíduos com perda auditiva foi $21,91 \mathrm{~dB}$ maior que 
o valor médio dos indivíduos com audição normal. Da mesma forma, o valor médio do LRSS foi 23,58 $\mathrm{dB}$ e do IPMRSS foi 28,42 maior no grupo de indivíduos com perda auditiva neurossensorial.

A variabilidade observada entre indivíduos também foi maior no grupo $B$, pois os graus de perda dos indivíduos avaliados nesta pesquisa foram de leve a moderadamente severo (Tabela 1). Porém, estas diferenças individuais não estão correlacionadas somente aos limiares auditivos.

Indivíduos com perda neurossensorial podem apresentar uma gama de resultados no reconhecimento de fala, que podem ser determinados pelo grau de perda, configuração e extensão do comprometimento auditivo ${ }^{1}$, mas os resultados também, sofrem influência de outros fatores, como a inteligência, memória, idade, nível de estresse, motivação do paciente, entre outros ${ }^{7,17}$.

Ao comparar os resultados obtidos no grupo de indivíduos normo-ouvintes e no grupo com perda auditiva, observou-se que existe diferença estatisticamente significante em todas as variáveis analisadas (Tabela 1).

As curvas logoaudiométricas resultantes das avaliações realizadas nos dois grupos (Figura 2) demonstram o desempenho dos indivíduos na ordenada (eixo y) e a intensidade de apresentação das sentenças, expressa em dB (A) NPS, na abscissa (eixo $x$ ).

Observa-se que a curva logoaudiométrica do grupo de indivíduos com perda auditiva neurossensorial mostrou-se deslocada em níveis de apresentação mais altos, cerca de $20 \mathrm{~dB}$ acima na abscissa, em relação à curva dos indivíduos com audição normal.

A melhora no desempenho à medida que a intensidade aumenta, também não é tão íngreme, quanto no grupo com audição normal.

A inclinação mais gradual nas curvas logoaudiométricas de indivíduos com perda auditiva está correlacionada ao fato desta população ser menos homogênea do que a população com audição normal, no que se refere ao reconhecimento de fala ${ }^{1}$.

Estes resultados estão de acordo com os encontrados na literatura que mostra que as diferenças encontradas entre o desempenho de reconhecimento de fala de pessoas com audição normal e com perda auditiva neurossensorial se deve à diferença de sensibilidade ao longo das frequências, que é característica da perda auditiva neurossensorial ${ }^{1,15}$.

Na literatura, são encontrados estudos que descrevem limiares logoaudiométricos pesquisados no silêncio, com diferentes tipos de estímulos (sílabas, palavras, sentenças com ou sem sentido, etc.), através de fones ou em campo livre, em indivíduos com audição normal e/ou perda auditiva e sua correlação com os limiares tonais encontrados na avaliação audiológica de rotina 1,3,5-8,11,14,15,18,19.

Porém, não foram encontrados na literatura, estudos realizando uma análise semelhante à feita neste estudo, no qual se busca avaliar o indivíduo em uma situação semelhante às encontradas na vida diária e relata seu desempenho em tarefas de reconhecimento de fala por meio de curvas logoaudiométricas, as quais se mostram uma ferramenta facilmente disponível e potencialmente útil, mas muito negligenciada atualmente nas pesquisas audiológicas e na audiologia clínica ${ }^{19}$.

A execução deste estudo permite, ainda, fazer um comentário a respeito da metodologia empregada na pesquisa dos máximos índices de reconhecimento, a fim de que sua reprodução em novas pesquisas ou sua utilização na prática clínica seja mais adequada.

Sugere-se que, ao realizar a pesquisa dos índices máximos em indivíduos com audição normal, primeiramente, sejam somados $4 \mathrm{~dB}$ ao valor encontrado na pesquisa do LRSS. Nesta intensidade, uma lista de sentenças é apresentada. Não ocorrendo o máximo índice (100\%), a intensidade pode ser aumentada em $2 \mathrm{~dB}$ e uma nova lista apresentada. Esse procedimento pode ser repetido até o indivíduo reconhecer todas as sentenças de uma lista. Se o pesquisador sentir necessidade de verificar se o indivíduo não teria o mesmo desempenho em uma intensidade menor, $1 \mathrm{~dB}$ pode ser subtraído da intensidade onde se obteve o índice de $100 \%$ de reconhecimento, e uma nova lista deve ser apresentada.

Quando a pesquisa dos índices máximos for realizada em indivíduos com perda auditiva, os acréscimos de intensidade entre uma lista e outra, podem ser dados de $4 \mathrm{em} 4 \mathrm{~dB}$ até que o indivíduo reconheça $100 \%$ das sentenças de uma lista. Para confirmar o máximo índice, o pesquisador pode subtrair 2 ou $1 \mathrm{~dB}$ e verificar se houve ou não mudança no índice.

Estas estratégias permitem a obtenção de resultados mais precisos na pesquisa dos máximos índices de reconhecimento.

Este estudo também permite sugerir que, para a pesquisa da real condição de comunicação de um indivíduo, devem-se introduzir na rotina clínica testes que utilizem sentenças como estímulo.

Destaca-se a importância da utilização dos dados obtidos neste estudo na rotina clínica, pois o conhecimento dos valores aqui apresentados pode contribuir para que o audiologista tenha maiores possibilidades de dimensionar a dificuldade de inteligibilidade de fala de seu paciente. 


\section{CONCLUSÃO}

Foram traçadas as curvas logoaudiométricas para os grupos estudados, a partir das avaliações realizadas no silêncio e em campo livre com o teste LSP.

Nos indivíduos normo-ouvintes (grupo A), os valores médios encontrados para o LDV, o LRSS e IPMRSS foram, respectivamente: 16,47; 23,77 e $31,23 \mathrm{~dB}(\mathrm{~A})$ NPS.

No grupo de indivíduos com perda auditiva neurossensorial (grupo B) os valores médios encontrados para o LDV, o LRSS e o IPMRSS foram, respectivamente: 38,$38 ; 50,35$ e $59,65 \mathrm{~dB}$ (A) NPS.

Foi encontrada diferença estatisticamente significante entre os resultados obtidos no grupo $A$ e no grupo B.

Observou-se que cada $1 \mathrm{~dB}$ de variação na intensidade de apresentação das sentenças, ocasionou uma mudança de $6,77 \%$ no reconhecimento de fala para os indivíduos normo-ouvintes e de $4,70 \%$ e no grupo de indivíduos com perda auditiva.

\section{ABSTRACT}

Purpose: to determine the Performance-Intensity function for sentences in a group of individuals with normal hearing and a group of individuals with sensorineural hearing loss, through the Portuguese Sentence List test (PSL), and compare the results obtained in these groups. Methods: the PSL test was used to determine voice detection threshold (VDT), recognition sentences in silence threshold (RSST) and maximum percent indexes of sentences recognition in silence (MPISRS), in a group of individuals with normal hearing (group $A$ ) and a group of individuals with sensorineural hearing loss (group B). Results: group A was made of 30 subjects, 19 female and 11 male, from 18 to 40 -year old. In group B, 15 subjects were female and 14 were male, from 23 to 81 -year old. In group A, were found average values of $16.47 \mathrm{~dB}$ for the VDT, $23.77 \mathrm{~dB}$ for the RSST and $31.23 \mathrm{~dB}$ for the MPISRS. In group B, were found values of $38.38 \mathrm{~dB}$ for the VDT, $50.35 \mathrm{~dB}$ for the RSST and $59.65 \mathrm{~dB}$ for the MPISRS. The statistical analysis showed statistically significant differences between the groups. Conclusion: performance-intensity functions for sentences were obtained with the PSL test. The results showed that each $1 \mathrm{~dB}$ of variation in intensity for sentence presentation represented a change of $6.77 \%$ in speech recognition for normal hearing individuals and $4.70 \%$ in a group of individuals with hearing loss.

KEYWORDS: Hearing; Speech; Speech Discrimination Tests; Speech Intelligibility; Speech Perception

\section{REFERÊNCIAS}

1. Wilson $\mathrm{RH}$, Strouse AL. Audiometria com estímulos de fala. In: Musiek FE, Rintelmann WF. Perspectivas atuais em avaliação auditiva. São Paulo: Manole; 2001. p. 21-56.

2. Henriques MO, Miranda EC, Costa MJ. Limiares de reconhecimento de sentenças no ruído, em campo livre: valores de referência para adultos normo-ouvintes. Rev Bras Otorrinolaringol. 2008; 74(2):188-92.

3. Costa MJ. Lista de sentenças em português: apresentação e estratégias de aplicação na audiologia. Santa Maria: Pallotti; 1998.

4. Bronkhorst AW, Plomp R. A clinical test for the assessment of binaural speech perception in noise. Audiology. 1990; 29(5):275-85.

5. Dubno JR, Ahlstrom JB, Horwitz AR. Binaural advantage for younger and older adults with normal hearing. J Speech Lang Hear Res. 2008; 51(2):539-56.

6. Freitas CD, Lopes LFD, Costa MJ. Confiabilidade dos limiares de reconhecimento de sentenças no silêncio e no ruído. Rev Bras Otorrinolaringol. 2005; 71(5):624-30.

7. Miranda EC, Costa MJ. Reconhecimento de sentenças no silêncio e no ruído de indivíduos jovens adultos normo-ouvintes em campo livre. Fono Atual. 2006; 8(35):4-12.

8. Aurélio NHS, Becker KT, Padilha C, Santos SN, Petry T, Costa MJ. Limiares de reconhecimento de sentenças no silêncio em campo livre versus limiares tonais em fone em indivíduos com perda auditiva coclear. Rev. CEFAC. 2008; 10(3):378-84. dx.doi.org/10.1590/S1516-18462008000300013

9. Silman S, Silverman CA. Auditory diagnosis, principles and applications. London: Singular Publishing Group; 1991. 
10. Boothroyd A. Speech perception, sensorineural hearing loss and hearing aid. In: Studevaker G, Hochberg I. Acoustical factors affecting hearing aid performance. Boston: Allyn \& Bacon; 1993. p. 277-99.

11. Mendel LL. Objective and subjective hearing aid assessment outcomes. Am J Audiol. 2007; 16(2):118-29.

12. Levitt $H$, Rabiner LR. Use of a sequential strategy in intelligibility testing. J Acoust Soc Am. 1967; 42(3):609-12.

13. Weisstein EW. Making mathWorld. Matemat J. 2007; 10(3):474-88.

14. Ribas A, Klagenberg k, Rosa M, Zeigelboim B, Martinsbassetto J. Comparação dos resultados do limiar de detectabilidade de voz por meio de material gravado e a viva voz. Rev. CEFAC. 2008; 10(4):5927. dx.doi.org/10.1590/S1516-18462008000400020
15. Osterne FJV. Limiares logoaudiométricos para palavras e sentenças. In: Pereira LD, Schochat E. Processamento auditivo central: manual de avaliação. São Paulo: Lovise; 1997. p. 43-7.

16. McFarland $\mathrm{WH}$. Próteses auditivas e compreensão da fala. In: Almeida K, lório MCM. Próteses auditivas: fundamentos teóricos e aplicações clínicas. 2. ed. São Paulo: Lovise; 2003. p. 17-34.

17. Caporali SA, Silva JA. Reconhecimento de fala no ruído em jovens e idosos com perda auditiva. Rev Bras Otorrinolaringol. 2004; 70(4):525-32.

18. Soncini F, Costa MJ, Oliveira TMT, Lopes LF. Correlação entre limiares de reconhecimento de sentenças no silêncio e limiares tonais. Rev Bras Otorrinolaringol. 2003; 69(5):672-7.

19. Boothroyd A. The performance/intensity function: an underused resource. Ear Hear. 2008; 29(4):479-91.

DOI: 10.1590/S1516-18462010005000004

RECEBIDO EM: 20/08/2009

ACEITO EM: 02/10/2009

Endereço para correspondência:

Nilvia Herondina Soares Aurélio

Rua Venâncio Aires, 454-A

Santa Maria - RS

CEP: 97010-001

E-mail: nilviaaurelio@yahoo.com.br 